\title{
Dark Hadrons as Dynamical Dark Matter
}

\author{
Keith R. Dienes* \\ Department of Physics, University of Arizona, Tucson, AZ 85721 USA \\ Department of Physics, University of Maryland, College Park, MD 20742 USA \\ E-mail: dienes@email.arizona.edu
}

\section{Fei Huang}

Department of Physics, University of Arizona, Tucson, AZ 85721 USA

E-mail: huangfei@email.arizona.edu

\section{Shufang Su}

Department of Physics, University of Arizona, Tucson, AZ 85721 USA

E-mail: shufang@email.arizona.edu

\section{Brooks Thomas}

Department of Physics, Lafayette College, Easton, PA 18042 USA

E-mail: thomasbdelafayette.edu

\begin{abstract}
Dynamical Dark Matter (DDM) is an alternative framework for dark-matter physics in which the dark sector comprises a vast ensemble of particle species whose Standard-Model decay widths are balanced against their cosmological abundances. Previous studies of this framework have focused on a particular class of DDM ensembles in which the density of dark states scales roughly as a polynomial of the mass. In this talk, by contrast, we discuss the properties of DDM ensembles in which the density of dark states grows exponentially with mass, as appropriate if the dark-sector constituents are the "hadronic" resonances associated with the confining phase of a stronglycoupled dark sector. We find that rather generic cosmological constraints introduce non-trivial correlations between the mass scales, lifetimes, and abundance distributions associated with these DDM ensembles. For example, we find that such DDM ensembles can exhibit energy scales ranging from the $\mathrm{GeV}$ scale all the way to the Planck scale, but that the total present-day cosmological abundance of the dark sector must be spread across an increasing number of different states in the ensemble as these energy scales are dialed from the Planck scale down to the GeV scale. This talk reports on work originally presented in Ref. [1].
\end{abstract}

XIII Quark Confinement and the Hadron Spectrum - Confinement 2018

31 July - 6 August 2018

Maynooth University, Ireland

\footnotetext{
* Speaker.
} 


\section{Introduction}

Dynamical Dark Matter (DDM) [2,3] is an alternative framework for dark-matter physics in which dark-matter stability is not required. Instead, the dark sector within the DDM framework comprises a vast ensemble of individual constituent particles exhibiting a variety of different masses, lifetimes, and cosmological abundances such that those ensemble constituents with shorter lifetimes must have smaller cosmological abundances, while states with longer lifetimes may have larger cosmological abundances. As a result, the dark sector in such a scenario is dynamic: states in the dark sector are continually decaying into visible-sector states throughout the evolution of the universe - not just in previous epochs but even at the present time and into the future. Many methods have been developed for testing this framework, spanning from collider signatures $[4,5]$ to signatures in direct-detection [6] and indirect-detection $[7,8,9]$ experiments.

Many of the phenomenological properties of these DDM ensembles depend on the manner in which the lifetimes and abundances of ensemble constituents scale with respect to each other. One general class of DDM ensembles are those whose constituents are the Kaluza-Klein (KK) modes of a gauge-neutral bulk field in a theory with extra spacetime dimensions in which cosmological abundances are established through misalignment production [2]. Indeed, explicit realizations of DDM ensembles of this type have been constructed $[3,10]$. In such ensembles, the cosmological abundance of each component scales as a power of the lifetime of that component. Likewise, the density of states within such ensembles is either insensitive to mass or scales roughly as a polynomial function of mass across the ensemble. Polynomial scaling relations also emerge in other (purely four-dimensional) contexts as well. For example, under certain circumstances, thermal freeze-out mechanisms for abundance generation can also lead to appropriate polynomial inverse scaling relations between lifetimes and abundances [11]. In fact, such inverse scaling relations can even emerge statistically in contexts in which the dynamics underlying the dark sector is essentially random [12]. For these reasons, most phenomenological studies of the DDM framework have focused on ensembles exhibiting polynomial scaling relationships.

There are, however, other well-motivated theoretical constructions which do not give rise to dark sectors with polynomial scaling relations. One example is a dark sector consisting of a set of fermions (dark "quarks") charged under a non-Abelian gauge group $G$ which becomes confining below some critical temperature $T_{c}$. At temperatures $T \lesssim T_{c}$, when the theory is in the confining phase, the physical degrees of freedom are composite states (dark "hadrons"). Such ensembles have two fundamental properties:

- mass distributions which follow linear Regge trajectories (i.e., $\alpha^{\prime} M_{n}^{2} \sim n$ where $\alpha^{\prime}$ is a corresponding Regge slope), and

- exponentially growing ("Hagedorn-like") degeneracies of states (i.e., $g_{n} \sim e^{\sqrt{n}} \sim e^{\sqrt{\alpha^{\prime}} M_{n}}$ ).

These features - especially the appearance of an exponential scaling of the state degeneracies with mass - represent a behavior which is markedly different from that exhibited by DDM ensembles with polynomial scaling relations. In this paper, we shall study the generic properties of DDM ensembles which exhibit the two features itemized above. 


\section{Our framework: Fundamental assumptions}

As discussed above, the first feature that we shall assume of our hadronic dark sector is a mass spectrum consistent with the existence of Regge trajectories. The existence of such trajectories follows directly from our assumption that our dark-sector bound states can be modeled by dark quarks connected by the confining flux tube associated with a strong, attractive, dark-sector interaction. Taking meson-like configurations as our guide, it can easily be shown that the mass $M_{n}$ associated with a relativistic rotating flux tube scales with the corresponding total angular momentum $n$ as $n \sim \alpha^{\prime} M_{n}^{2}$, where $\alpha^{\prime}$ is the so-called Regge slope. In the visible sector, this successfully describes the so-called leading Regge trajectory of the observed mesons, with $\alpha^{\prime} \sim 1(\mathrm{GeV})^{-2}$ appropriate for QCD. Moreover, there also exist subleading (parallel) Regge trajectories of observed mesons which have the same Regge slope but different intercepts: $n \sim \alpha^{\prime} M_{n}^{2}+\alpha_{0}$. We shall thus assume that the states of our dark "hadronic" DDM ensemble have discrete positive masses $M_{n}$

$$
M_{n}^{2}=n M_{s}^{2}+M_{0}^{2} .
$$

where the index $n$ labels our states in order of increasing mass. Here $M_{s} \equiv 1 / \sqrt{\alpha^{\prime}}$ is the corresponding "string scale", while $M_{0}$ represents the mass of the lightest "hadronic" constituent in the DDM ensemble. We shall avoid making any further assumptions about the nature of the dark sector by treating both $M_{s}$ and $M_{0}$ as free parameters to be eventually constrained by cosmological data.

The second generic feature associated with hadronic spectroscopy is the well-known exponential rise in the degeneracies of hadrons as a function of mass: $g_{n} \sim e^{\sqrt{n}}$. In general, we can understand this behavior as follows. If we model our dark hadrons as dark quarks connected by flux tubes, the degeneracy $g_{n}$ of hadronic states at any mass level $n$ can be written as the product of two contributions: one factor $\kappa$ representing a multiplicity of states due to the degrees of freedom associated with the dark quarks (such as the different possible configurations of quantities like spin and flavor), and a second factor $\hat{g}_{n}$ representing the multiplicity of dark states due to the degrees of freedom associated with the flux tube. We thus have

$$
g_{n} \approx \kappa \hat{g}_{n} .
$$

While the constant $\kappa$ is independent of the mass level $n$, the remaining degeneracy factor $\hat{g}_{n}$ counts the rapidly increasing number of ways in which a state of given total energy $n$ can be realized as a combination of the vibrational, rotational, and internal excitations of the different harmonic oscillators which together describe the quantized flux tube. It is this quantity which grows exponentially with mass, and in this work we shall generally take $\hat{g}_{n}$ for all $n \geq 1$ to be given as [1]

$$
\begin{aligned}
\hat{g}_{n} & =2 \pi\left(\frac{16 \pi^{2} n}{C^{2}}-1\right)^{\frac{1}{4}-B} I_{\left|2 B-\frac{1}{2}\right|}\left(C \sqrt{n-\frac{C^{2}}{16 \pi^{2}}}\right) \\
& \approx \frac{1}{\sqrt{2}}\left(\frac{C}{4 \pi}\right)^{2 B-1} n^{-B} e^{C \sqrt{n}} \quad \text { as } n \rightarrow \infty
\end{aligned}
$$

where $B$ and $C$ are free parameters which describe the physics of the flux tube. In the first line of Eq. (2.3), $I_{v}(z)$ denotes the modified Bessel function of the first kind of order $v$. Use of the 
approximation $I_{v}(z) \approx e^{z} / \sqrt{2 \pi z}$ for $z \gg 1$ then reproduces the result on the second line of Eq. (2.3). For $n=0$, by contrast, we shall define $\hat{g}_{0} \equiv 1$, representing the unique ground state of our flux tube.

Clearly the most salient property of the expression in Eq. (2.3) is that it rises exponentially with $\sqrt{n}$, or equivalently with the mass $M_{n}$ of the corresponding state. This is ultimately because the degrees of freedom associated with our flux tube include so-called oscillator excitations representing the internal fluctuations of the flux tube itself. It is the multitude of oscillator excitations which give rise to the exponentially growing degeneracies.

Thus far, we have introduced four parameters to describe our dark "hadron" DDM ensemble: $M_{s}, M_{0}, B$, and $C$. The first two parameters have immediate interpretations: $M_{0}$ is the mass of the lightest state in the DDM ensemble, while $M_{s}$ parametrizes the splitting between the states. However $B$ and $C$ can also be understood physically. In general, we can describe the dynamics of any flux tube propagating in a spacetime of effective dimensionality $D$ through an action whose leading behavior is described through the Polyakov action:

$$
S \sim M_{s}^{2} \int d^{2} \sigma \sum_{i=1}^{D_{\perp}}\left(\frac{\partial}{\partial \sigma^{\alpha}} X^{i}\right)\left(\frac{\partial}{\partial \sigma_{\alpha}} X^{i}\right)+\ldots
$$

Here the $D_{\perp}$ embedding functions $X^{i}\left(\sigma_{1}, \sigma_{2}\right), i=1, . ., D_{\perp}$, are the transverse spacetime locations of any point on the flux-tube surface with coordinates $\left(\sigma_{1}, \sigma_{2}\right)$. Minimizing this action is classically equivalent to minimizing the area of the flux-tube worldsheet. In general, one may parametrize the total numbers of degrees of freedom for such an action in terms of a quantity known as the central charge $c$, where $c_{\text {int }}=c-D_{\perp}$ parametrizes the number of degrees of freedom associated with whatever extra terms might appear in the action beyond those explicitly shown in Eq. (2.4). One then finds a relatively straightforward connection between the parameters $(B, C)$ appearing in our state degeneracies and the parameters $\left(D_{\perp}, c\right)$ of our underlying flux-tube theory [1]:

$$
\begin{aligned}
& B=\frac{1}{4}\left(3+D_{\perp}\right) \\
& C=\pi \sqrt{2 c / 3} .
\end{aligned}
$$

We shall allow $D$ (and by extension $D_{\perp}$ ) to be arbitrary in order to account for a range of general theoretical possibilities discussed in Ref. [1]. Likewise, the parameters $B$ and $C$ are subject to certain self-consistency constraints which are also discussed in Ref. [1].

Finally, we must make certain assumptions about the cosmological abundances $\Omega_{n}$ and decay widths $\Gamma_{n}$ that are associated with these ensemble states. We have been imagining that these dark "hadrons" emerge as the result of a dark-sector confining phase transition triggered by the strong interactions of some dark-sector gauge group $G$. This phase transition occurs when the temperature $T$ in the dark sector drops below the critical temperature $T_{c}$ associated with this phase transition. This event marks the time $t_{c}$ at which the primordial abundances of our individual hadrons are established. Moreover, it is reasonable to assume that residual $G$ interactions establish thermal equilibrium among these hadrons at $T \sim T_{c}$. Thus, the primordial abundances $\Omega_{n}$ of our hadrons can be assumed to follow a Boltzmann distribution at $t=t_{c}$ :

$$
\Omega_{n}\left(t_{c}\right) \equiv \frac{\rho_{n}\left(t_{c}\right)}{\rho_{\text {crit }}\left(t_{c}\right)}=\frac{1}{3 \widetilde{M}_{P}^{2} H\left(t_{c}\right)^{2}} \int \frac{d^{3} \mathbf{p}}{(2 \pi)^{3}} E_{\mathbf{p}} e^{-E_{\mathbf{p}} / T_{c}}
$$


where $E_{\mathbf{p}} \equiv \sqrt{\mathbf{p} \cdot \mathbf{p}+M_{n}^{2}}$ and $\rho_{\text {crit }}(t) \equiv 3 \widetilde{M}_{P}^{2} H(t)^{2}$ where $\widetilde{M}_{P} \equiv M_{P} / \sqrt{8 \pi}=1 / \sqrt{8 \pi G_{N}}$ is the reduced Planck mass and $H(t)$ the Hubble parameter. We shall require that $T_{c} / M_{s} \leq 1 / C$ in order to have a finite total abundance for the ensemble as a whole. We shall also henceforth assume that $T_{c}, M_{s}, M_{0}>T_{\mathrm{MRE}}$ where $t_{\mathrm{MRE}}$ and $T_{\mathrm{MRE}}$ are the time and temperature associated with matterradiation equality. This assumption, which parallels what occurs for the hadrons of the visible sector, ensures that our abundances $\Omega_{n}(t)$ are established during the radiation-dominated era prior to matter-radiation equality and that all ensemble constituents have become effectively non-relativistic by $t_{\mathrm{MRE}}$. Of course, the expression in Eq. (2.6) describes the abundance of each constituent at the time $t_{c}$. This quantity then evolves in time due to the Hubble expansion of the universe and the possible decay of the constituent.

Turning now to the decays of our ensemble constituents, we immediately recognize that there are two kinds of decays that might be relevant. First, there are decays within the dark sector, wherein a heavy dark state decays to lighter dark states within the ensemble. Such intra-ensemble decays depend on the dark-sector coupling and could lead to highly non-trivial phase-space distributions for the lightest remaining dark-matter states. This could potentially alter cosmological structure formation and the resulting matter power spectrum. By contrast, there can be decays out of the dark sector and directly into Standard-Model (visible) states. Such decays depend on the coupling between the dark and visible sectors, and could potentially cause difficulties with BBN and/or CMB constraints, and/or leave undesirable imprints in photon/X-ray spectra.

In this paper, we shall assume that the net injection of energy density in the form of radiation from dark-sector decays has a negligible effect on the total radiation-energy density of the universe. Second, we shall further assume that the contribution to the total decay width $\Gamma_{n}$ of each ensemble constituent from intra-ensemble decays is negligible. In other words, we shall assume that $\Gamma_{n}$ is dominated by decays to visible-sector final states which do not include lighter ensemble constituents. We shall discuss this assumption further in the Conclusions. Third, we shall assume that all states at a given mass level $n$ share a common decay width $\Gamma_{n}$, and that this width scales with $n$ across our dark-hadron ensemble according to

$$
\Gamma_{n}=\Gamma_{0}\left(\frac{M_{n}}{M_{0}}\right)^{\xi}
$$

where $M_{n}$ are the dark-hadron masses in Eq. (2.1) and where $\Gamma_{0}$ and the scaling exponent $\xi>0$ are taken to be additional free parameters. Finally, for simplicity, we shall imagine that all states with lifetimes $\tau_{n} \equiv 1 / \Gamma_{n}$ indeed actually decay at $t=\tau_{n}$.

\section{Cosmological constraints on the dark-hadron ensemble}

We now describe three quantities which ultimately play very important roles in characterizing and constraining such dark-hadron DDM ensembles.

The first property of a given dark-hadron DDM ensemble that shall concern us is its total abundance $\Omega_{\text {tot }}(t) \equiv \sum_{n=0}^{\infty} g_{n} \Omega_{n}(t)$. However, we are also interested in tracking the distribution of this total abundance among the individual ensemble constituents. For this purpose we shall also 
define [2] a so-called "modified tower fraction" $0 \leq \eta(t)<1$ as

$$
\eta(t) \equiv 1-\frac{\max _{n}\left\{\widehat{\Omega}_{n}(t)\right\}}{\Omega_{\text {tot }}(t)}
$$

where $\widehat{\Omega}_{n}(t) \equiv g_{n} \Omega_{n}(t)$ is the aggregate cosmological abundance arising from all states at oscillator level $n$. Thus $\eta \approx 0$ signifies a dark sector resembling traditional single-component dark matter (where the "single component" corresponds to the degenerate states at the dominant mass level), while $\eta>0$ indicates (and quantifies) a "DDM-like" departure from this traditional scenario, one in which states at all mass levels contribute non-trivially in shaping the properties of the dark sector.

Finally, the third important quantity which can be taken to characterize our dark sector is the so-called equation-of-state parameter $w$. For a single-component dark sector, this is the ratio between the pressure $p$ and energy density $\rho$ of the dark component: $p=w \rho$. However, we are dealing here with a multi-component dark sector in which each component has its own individual lifetime and abundance. As a result, the total energy density and pressure associated with our dark sector will generally experience a rather non-trivial time dependence which causes our ensemble as a whole to behave collectively as if it had a non-trivial $w$ - even if each individual component is taken to be pure matter with $w=0$. To describe these collective effects, we therefore define [2] an effective equation-of-state parameter $w_{\text {eff }}(t)$ which describes the behavior of our ensemble as a single collective entity:

$$
w_{\mathrm{eff}}(t) \equiv-\left(\frac{1}{3 H} \frac{d \log \rho_{\mathrm{tot}}}{d t}+1\right)=-\frac{t}{2 \Omega_{\mathrm{tot}}} \frac{d \Omega_{\mathrm{tot}}(t)}{d t} .
$$

Here $H$ is the Hubble parameter and $\rho_{\text {tot }}=3 \widetilde{M}_{P} H^{2} \Omega_{\text {tot }}$ is the total energy density of the ensemble. Note that the definition in Eq. (3.2) is nothing but the usual definition of $w$ prior to any assumptions of dark-sector minimality. By contrast, the second equality applies only during the present matter-dominated epoch, within which $H(t) \approx 2 /(3 t)$. Note that even though each of the individual components of our ensemble has been taken to be matter-like (with $w=0$ ), the collective equation-of-state parameter $w_{\text {eff }}(t)$ for our ensemble as a whole is positive, reflecting the fact that the ensemble as a whole is continually losing abundance as its individual components decay.

Given these quantities, we can impose certain "zeroth-order" cosmological/astrophysical constraints. These will enable us to obtain an initial sense of those parameter-space regions in which a DDM dark-hadron ensemble might have at least the potential of phenomenological viability.

First, we shall demand that $\Omega_{\text {tot }}\left(t_{\text {now }}\right)=\Omega_{\mathrm{CDM}} \approx 0.26$, a requirement predicated on the assumption that our dark-hadronic ensemble represents the totality of the dark sector. Second, we shall also constrain the the time-variation of $\Omega_{\text {tot }}(t)$ using information about the dark-matter abundance during different cosmological epochs. For example, CMB data provides information about the dark-matter abundance around the time of last scattering - i.e., at a redshift $z \approx 1100$, or equivalently a time of roughly $2.7 \times 10^{-5} t_{\text {now }}$. On the other hand, observational data on baryon acoustic oscillations and the relationship between luminosity and redshift for Type Ia supernovae provide information about $H(t)$ and the dark-energy abundance $\Omega_{\Lambda}$ at subsequent times, down to redshifts of around $z \approx 0.5$. Within the context of the $\Lambda \mathrm{CDM}$ cosmology, the agreement between these different measurements implies that the dark-matter abundance has not changed dramatically since the time of last scattering. In order be consistent with this result, we shall therefore demand that 
the total abundance of our DDM ensemble not vary by more than $5 \%$ between an early "look-back" time $t_{\mathrm{LB}}$ and today:

$$
\frac{\Omega(t)-\Omega\left(t_{\text {now }}\right)}{\Omega\left(t_{\text {now }}\right)} \leq 0.05 \text { for all } t_{\mathrm{LB}} \leq t \leq t_{\text {now }} .
$$

In what follows, we shall take $t_{\mathrm{LB}}=10^{-6} t_{\text {now }}$, comfortably before the recombination epoch. We can also place a bound on the rate of change of the total dark-matter abundance within the very recent past $-i . e$., for redshifts in the range $0 \lesssim z \lesssim 3$. Specifically, we shall impose an additional constraint on our effective equation-of-state parameter: $w_{\text {eff }}\left(t_{\text {now }}\right) \leq 0.05$. Finally, we shall demand that $M_{0} \gtrsim \mathscr{O}\left(10^{3}\right) T_{\mathrm{MRE}} \approx \mathscr{O}(\mathrm{keV})$ in order to satisfy BBN and structure-formation constraints.

We shall also make two simplifying assumptions. First, for concreteness, we shall restrict our attention to situations with $\xi=3$, corresponding to decay widths which scale as $\Gamma_{n} \sim M_{n}^{3} / \Lambda^{2}$, as appropriate for situations in which the decay products are extremely light compared to $M_{n}$. Such widths dominate the decays of our DDM constituents in scenarios in which our DDM ensemble is uncharged with respect to all Standard-Model symmetries, and in which intra-ensemble decays can be neglected. Likewise, we shall also make the simplifying assumption that $\kappa=1$ in Eq. (2.2). This restricts us to the bare "minimal" case in which we do not ascribe non-trivial degrees of freedom to our dark-sector quarks, and thereby focus exclusively on the ensemble of states generated by our infinite tower of hadronic resonances.

\section{Results}

We begin by adopting the "benchmark" values $(B, C)=(5 / 4,2 \pi / \sqrt{3})$ and define the dimensionless variables $r \equiv M_{0} / M_{s}$ and $s \equiv T_{c} / M_{s}$. We then find the results shown in Fig. 1. It is immediately clear from these results that there is a "sweet spot" within the $(r, s)$ parameter space, lying roughly within the range

$$
\text { "sweet spot": } \quad 1 \lesssim r \lesssim 6, \quad 0.05 \lesssim s \lesssim 0.18
$$

Within this region, all of the constraints discussed above are satisfied. Moreover, within this region we see that $M_{s}$ varies from the $\mathrm{keV} / \mathrm{MeV}$-range all the way to the Planck scale. Likewise, $\eta\left(t_{\text {now }}\right)$ varies through all of its possible values. This is therefore not only an allowed region, but one which is likely to be exceedingly rich in phenomenology. Indeed, within this region, we observe from the left panel of Fig. 1 that $\eta\left(t_{\text {now }}\right)$ increases if either $r$ or $s$ is increased, while $M_{s}$ increases in the former case but decreases in the latter. Likewise, from the right panel, we see that $M_{s}$ increases extremely rapidly as a function of $r / s$. Fujrthermore, increasing the value of $r / s$ while holding $r$ fixed tends to decrease the value of $\eta\left(t_{\text {now }}\right)$. Thus, for fixed $r$, we find that $M_{s}$ and $\eta\left(t_{\text {now }}\right)$ tend to vary inversely with respect to each other as functions of $r / s$, with our ensembles becoming less DDM-like at higher mass scales and more DDM-like at lower mass scales. All of these results can easily be understood physically [1].

We now examine the characteristics of the corresponding ensembles. As discussed above, the most relevant aggregate properties of any dark-sector ensemble are its total cosmological abundance $\Omega_{\text {tot }}(t)$ and its effective equation-of-state parameter $w_{\text {eff }}(t)$, both of which is generally timedependent. We can therefore examine how each of these quantities evolves with time for ensembles in and near our sweet spot. This information is shown in Fig. 2. In this figure, we consider 

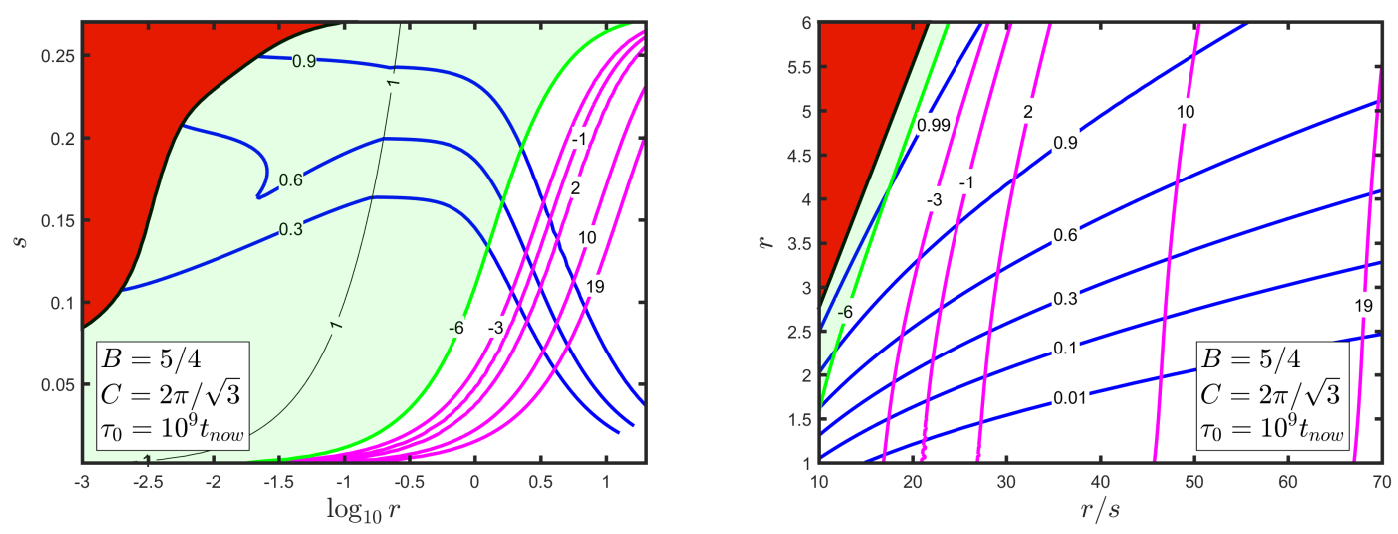

Figure 1: A survey of physics in the $(r, s)$ plane, with $B, C$, and $\tau_{0}$ set to the "benchmark" values shown. Left panel: The thin black line labeled ' 1 ' indicates the contour with $r / s=1$; this is thus the dividing line between the region in which the lightest state is relativistic (left of this line) versus non-relativistic (right of this line). The blue curves indicate contours of $\eta\left(t_{\text {now }}\right)$, while the magenta lines indicate contours of $M_{s}$ and are labelled by the value of $\log _{10}\left(M_{s} / \mathrm{GeV}\right)$. The red region is excluded by look-back and $w_{\text {eff }}$ constraints, while the pale green region is excluded by the constraint $M_{0} \gtrsim \mathscr{O}(\mathrm{keV})$ which is saturated along the single green contour. Increasing (decreasing) the value of $\tau_{0}$ does not affect the $M_{s}$ or $\eta\left(t_{\text {now }}\right)$ contours, and simply shifts the red exclusion region to the left (right). Right panel: Same as left panel, but with features plotted relative to $r$ and $r / s$. The entire region shown in this panel corresponds to the non-relativistic case.

a "benchmark" ensemble with $B=5 / 4, C=2 \pi / \sqrt{3}, r=3.5, s=3.5 / 30$, and $\tau_{0}=10^{9} t_{\text {now }}$, as well as nearby ensembles in which either $r$ (top row) or $s$ (bottom row) is varied. In each case, we plot the corresponding total cosmological abundance $\Omega_{\text {tot }}$ (left column) and equation-of-state parameter $w_{\text {eff }}$ (right column). Note that in each case the overall abundance is normalized through an appropriate choice of $M_{s}$ such that $\Omega\left(t_{\text {now }}\right)=\Omega_{\mathrm{CDM}} \approx 0.26$, as required.

In each panel of Fig. 2, the blue curve corresponds to our "benchmark" point. We therefore begin by focussing on these benchmark curves. The curve for $\Omega_{\text {tot }}(t)$ appears nearly constant at $\Omega_{\mathrm{CDM}} \approx 0.26$ for all of the cosmological history plotted (which we assume to have been matterdominated), including the present time $t_{\text {now }}$. Indeed, this behavior continues all the way into the future until $t \approx 10^{9} t_{\text {now }}$, at which point $\Omega_{\text {tot }}(t)$ begins to decline gently to $\Omega_{\text {tot }}=0$. This behavior is matched by $w_{\text {eff }}(t)$, which remains near zero for most its cosmological evolution before gently rising to $w_{\text {eff }}>0$ at $t \approx 10^{9} t_{\text {now }}$. This makes sense, since $w_{\text {eff }}(t)$ is proportional to the time-derivative of $\Omega_{\mathrm{tot}}(t)$. If this has been a traditional ensemble with a single dark-matter component whose decay we could model as essentially instantaneous (just as we are assuming for the individual components of our dark-matter ensembles), our curve for $\Omega_{\text {tot }}(t)$ would have been fixed precisely at its present value $\Omega_{\mathrm{CDM}} \approx 0.26$ over the entire range shown until suddenly dropping (essentially discontinuously) to $\Omega_{\text {tot }}=0$ when the single dark-matter particle decays at $t \approx 10^{9} t_{\text {now }}$. Likewise, $w_{\text {eff }}(t)$ would have been strictly fixed at $w_{\text {eff }}=0$ during the cosmological evolution. However, this is not a traditional dark-matter setup: this is a DDM ensemble in which the present-day cosmological abundance $\Omega_{\text {tot }}\left(t_{\text {now }}\right) \approx 0.26$ is spread across a relatively large number of individual components with different masses and different lifetimes. It is thus the continued, sequential decays of these different components which produce the softer, gentler drop in $\Omega_{\text {tot }}(t)$ as $t$ approaches $t \approx 10^{9} t_{\text {now }}$. 

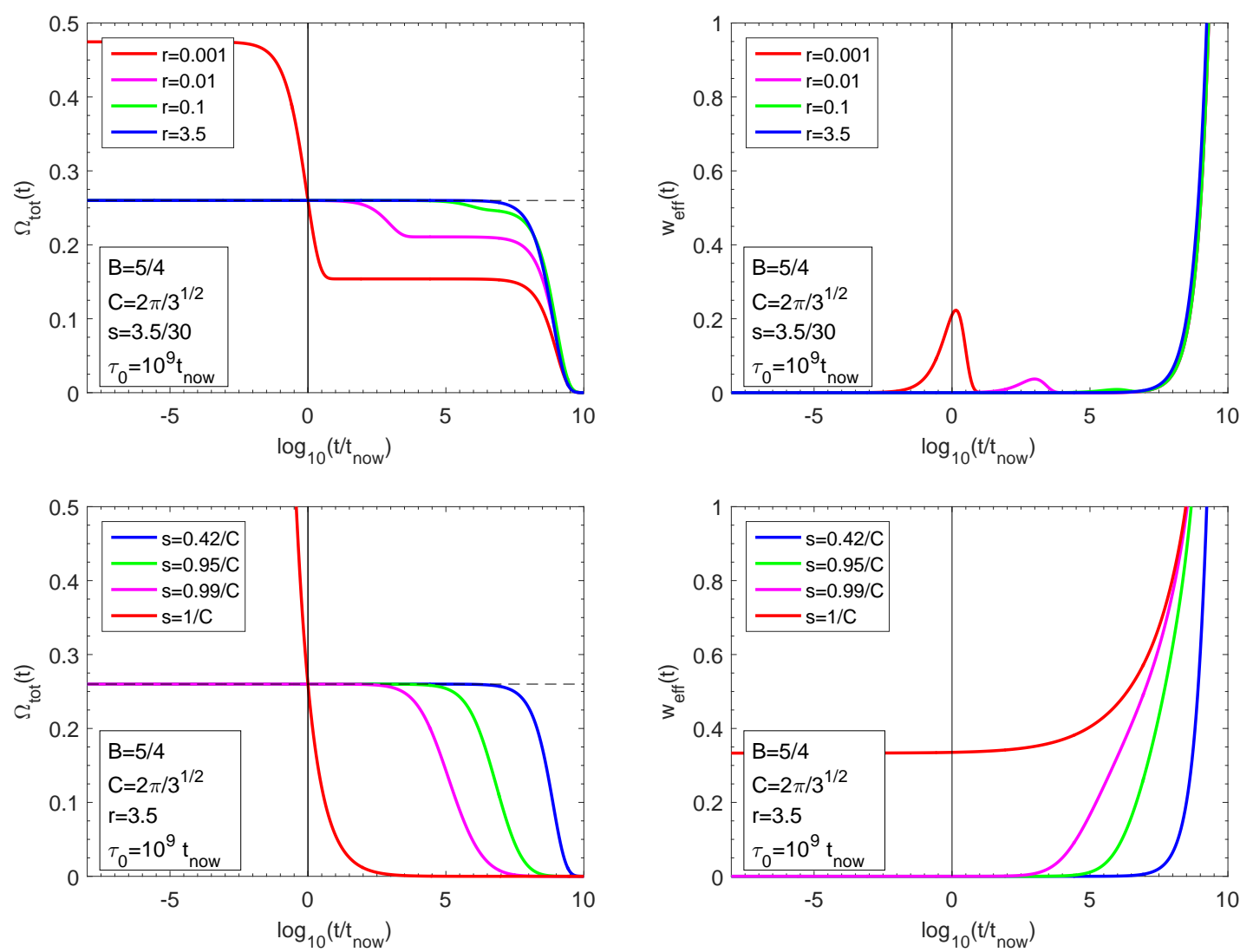

Figure 2: Total cosmological abundances $\Omega_{\text {tot }}$ (left column) and equation-of-state parameters $w_{\text {eff }}$ (right column) for our DDM ensembles, plotted as functions of time when all input variables are held fixed at their "benchmark" values except for $r$ (first row) and $s$ (third row). In all panels the blue curve corresponds to our "benchmark" point with $B=5 / 4, C=2 \pi / \sqrt{3}, r=3.5, s=3.5 / 30$, and $\tau_{0}=10^{9} t_{\text {now }}$, while the curves of other colors indicate departures away from this point. Note that, as expected, some variations away from the benchmark point violate our look-back, $w_{\text {eff }}$, or $M_{0}$ constraints. However, our internal self-consistency constraints are always satisfied, with $\Omega_{\text {tot }}\left(t_{\text {now }}\right)=\Omega_{\mathrm{CDM}} \approx 0.26$ in all cases.

We can also examine the individual constributions to the total present-day abundance $\Omega_{\text {tot }}$ from each individual mass level $n$. Our results are shown in Fig. 3. Fig. 3 consists of a sequence of dark-matter pie charts showing the relative contributions to $\Omega_{\text {tot }}\left(t_{\text {now }}\right)=\Omega_{\mathrm{CDM}} \approx 0.26$ from the lowest-lying states for $r=3.5$ (top row) and $r=4$ (bottom row), with $r / s=\{25,30,50,65\}$ across each row. Within each pie, we illustrate the corresponding collective abundances $\widehat{\Omega}_{n}\left(t_{\text {now }}\right)$ as separate slices, one for each value of $n$, while the numbers listed within each slice indicate the number of individual states $\hat{g}_{n}$ contributing at that mass level. For each pie chart we have also shown the corresponding values of $M_{0}, T_{c}$, and $M_{s}$. For these calculations we have used the input value $T_{\mathrm{MRE}}=0.7756 \mathrm{eV}$. We have also taken $g_{\mathrm{MRE}}=3.36$ and $g_{c}=\{10.75,61.75,106.75,106.75\}$, respectively, for $r / s=\{25,30,50,65\}$, where $g_{*}(T)$ tallies the number of effectively relativistic degrees of freedom driving the Hubble expansion at any temperature $T$, with $g_{\alpha} \equiv g_{*}\left(T_{\alpha}\right)$. We have also assumed our standard benchmark values $B=5 / 4, C=2 \pi / \sqrt{3}$, and $\tau_{0}=10^{9} t_{\text {now }}$. 

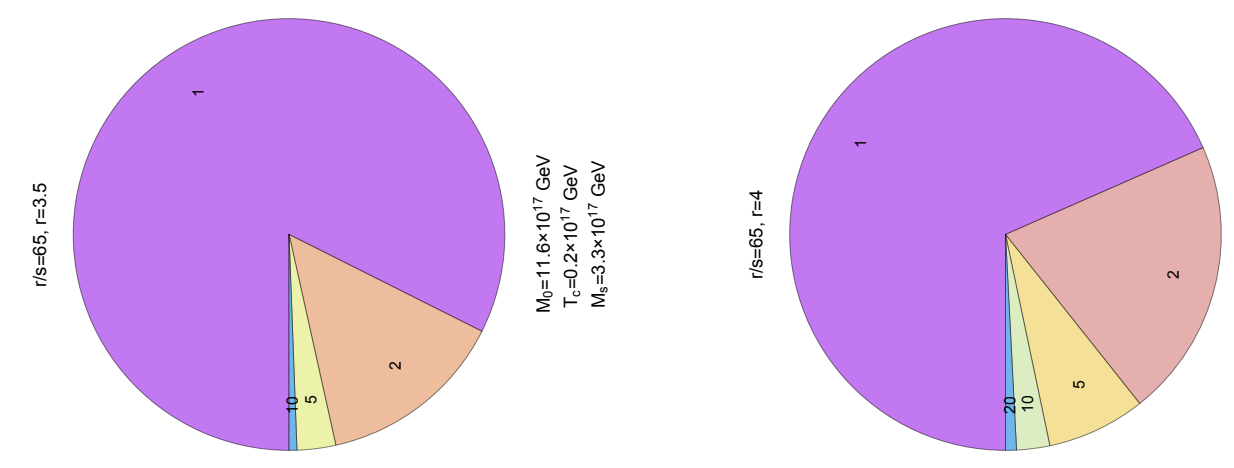

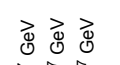

๗

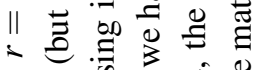

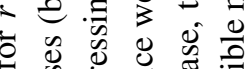

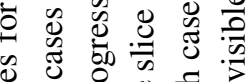
志

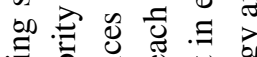

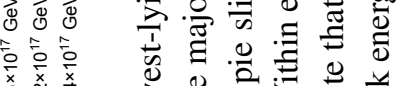

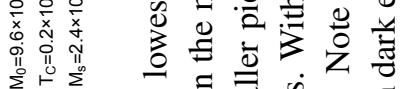

更

巳

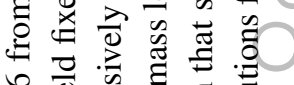

ำ

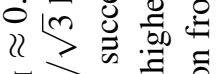
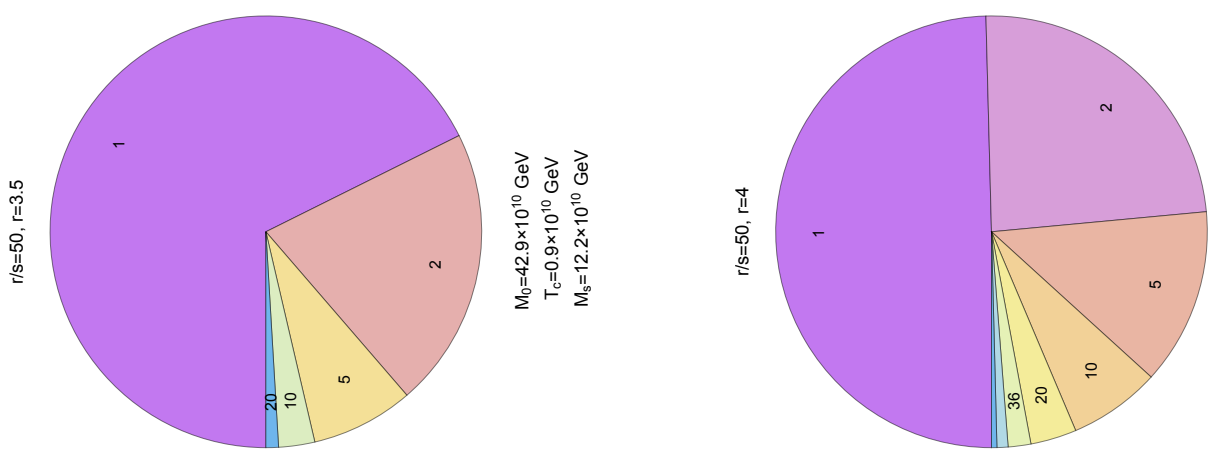

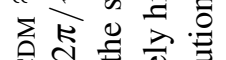

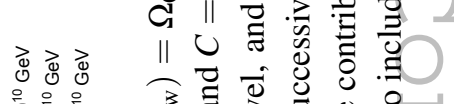

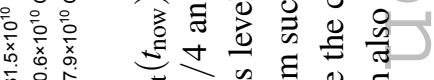

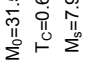
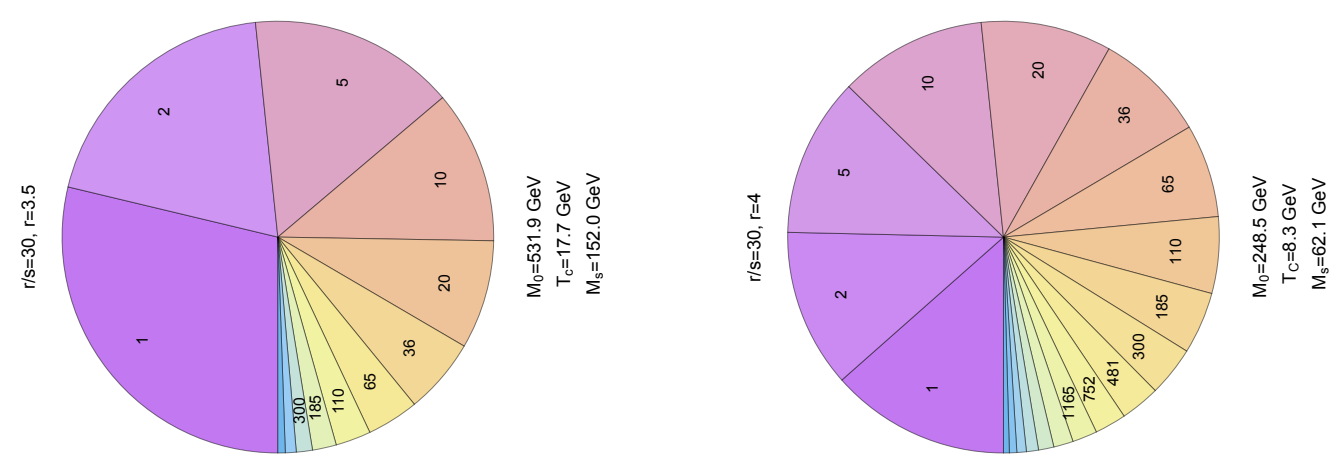

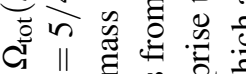

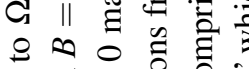

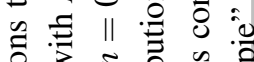

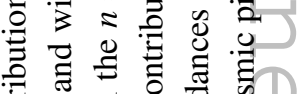

ฮ ठ उ

రิ

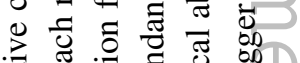

离

¿

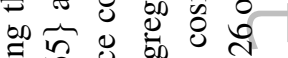

.

क

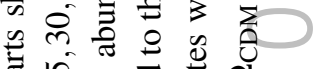

売

$\because 11$ 品
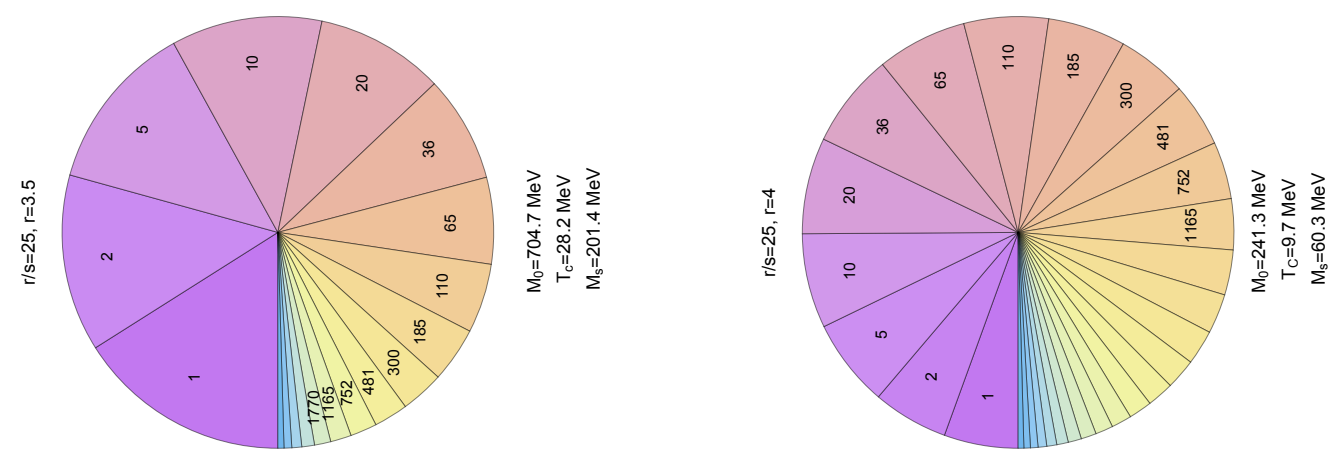

$\dot{0} \leqslant \tilde{0}$

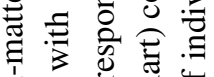

立

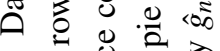

¿ छ

○.

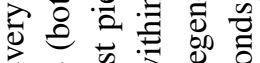

व + 范

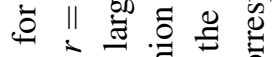

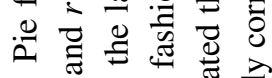

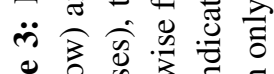

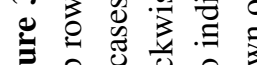

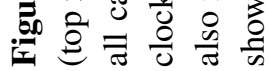


We see in general that increasing $r$ from 3.5 to 4.0 (i.e., passing from the top row of pie charts in Fig. 3 to the bottom row) has the net effect of shifting cosmological abundance away from the ground state, thereby increasing $\eta$ and generally making each pie slice smaller while simultaneously lowering the corresponding mass scales. This is in complete accord with the results shown in Fig. 1. Likewise, decreasing or increasing $r / s$ (i.e., moving left or right along either row) has the effect of increasing or decreasing $\eta$ while decreasing or increasing our corresponding mass scales. Indeed, we see that the variable $r / s$ allows us to interpolate between two extremes: traditional ensembles with high mass scales at large $r / s$ versus DDM-like ensembles with smaller mass scales at small $r / s$. We further observe that for sufficiently small $r / s$, the largest pie slice is no longer the $n=0$ slice (labelled ' 1 ' in each pie chart) — as $r / s$ decreases, this honor gradually shifts towards the pie slices corresponding to higher mass levels.

\section{Conclusions}

We have seen that a tremendous variety of DDM ensembles exist which have the two fundamental features outlined in the Introduction - Regge trajectories and exponentially rising degeneracies of states. These ensembles are consistent with our look-back and $w_{\text {eff }}$ constraints, and thus satisfy the zeroth-order constraints that may be imposed on such ensembles on the basis of their total energy densities and equations of state alone. We also observe an important feature, an inverse correlation between the tower fraction $\eta$ (which governs the extent to which our ensemble is truly DDM-like) and the magnitude of its underlying mass scales. Indeed, we have seen that while traditional ensembles typically have high corresponding mass scales, our ensembles become increasingly DDM-like for lower mass scales - all while remaining consistent with our look-back and $w_{\text {eff }}$ constraints. These observations will likely be an important guide and ingredient in any future attempts to build realistic dark-matter models of this type.

We are certainly not the first to consider dark-matter scenarios in which the dark matter is composite. Indeed, there exists a large literature of work in this direction. For example, within the context of traditional dark-matter models, it has been appreciated for some time that the darkmatter particle could be a composite state. For example, the lightest technibaryon in technicolor theories was long ago identified as a promising dark-matter candidate, and mechanisms were advanced by which this particle could be rendered sufficiently light so as to be phenomenologically viable. Other more exotic baryon-like composites have also been advanced as potential dark-matter candidates. A variety of scenarios in which a long-lived meson-like state which appears in the confining phase of a strongly-coupled hidden sector have been developed as well. These include scenarios in which the dark-matter particle is a pseudo-Nambu-Goldstone boson (PNGB) stabilized by a dark-sector analogue of flavor symmetry or $G$-parity, or alternatively by some other symmetry of the theory with no Standard-Model analogue. Scenarios in which the dark-matter candidate is not a PNGB, but rather a bound state of one heavy quark and one light quark, have also received recent attention primarily due to the non-standard direct-detection phenomenology to which they give rise, as have scenarios in which the dark-matter candidate is a bound state of heavy quarks alone. More general studies of composite hidden-sector theories which give rise to meson-like or baryon-like dark-matter candidates within different regions of parameter space have also been performed. Composite hidden-sector states consisting of non-Abelian gauge fields alone 
(so-called "glueball" states) have also long been recognized as promising dark-matter candidates. Indeed, such theories arise naturally in a variety string constructions, as well as in certain anomalymediated supersymmetry-breaking scenarios. In addition, the possibility that composite states in the dark sector could themselves form bound states (so-called "dark nuclei") has also been studied, as has the possibility that these nuclei themselves could combine to form dark "atoms" or even dark "molecules". There also exists many other ideas that have been pursued along these lines; a more complete discussion, along with appropriate references, is given in Ref. [1].

While all of these represent theoretically viable possibilities for the dark sector, the dark ensemble we have considered in this paper is unique for several important reasons. In traditional composite dark-matter models, it is usually a single bound state (usually the lightest bound state) which serves as the primary dark-matter candidate and which therefore carries the full dark-matter abundance $\Omega_{\mathrm{CDM}}$. While there may be several other dark states to which this bound state couples — and which may play a role in determining the abundance of the dark-matter candidate - it is nevertheless true that only one (or a few) composite states carry the dark-matter abundance $\Omega_{\mathrm{CDM}}$ and thereby play a significant role in dark-sector phenomenology. By contrast, within the DDM framework, the dark-matter abundance is potentially spread across a relatively large set of composite states with various masses and lifetimes. Thus the usual required stability of the traditional dark-matter candidate is not a required feature of the DDM ensemble, thereby allowing the associated dark-matter abundance $\Omega_{\mathrm{CDM}}(t)$ and dark-matter equation-of-state parameter $w_{\mathrm{eff}}(t)$ to vary with time - even during the current, matter-dominated era. Moreover, because the entire spectrum of composite states is potentially relevant for determining the properties of the dark sector within the DDM framework, features such as the existence of Regge trajectories and a Hagedorn-like exponential growth in the particle degeneracy — features which are cornerstones of strongly-coupled theories - are now relevant for determining dark-sector phenomenology.

Throughout this work, we have essentially assumed that our strongly-coupled dark hadrons decay primarily to states in the visible sector, rather than to lighter hadronic states within the DDM ensemble (and even those lying along the same Regge trajectory). At first glance, this might seem to be in conflict with our original supposition that the interactions governing the dynamics of the dark sector are sufficiently strong so as to produce hadron-like bound states in the first place. However, no conflict actually exists. ${ }^{1}$ In a nutshell, this is because the parameters that govern the binding of the dark quarks into dark hadrons are independent of the parameters that govern the intra-ensemble decay widths. In particular, the intra-ensemble decay widths of a strongly interacting theory depend not only on the strong coupling (related to string tension and/or the binding potential), but also on the masses of the dark quarks (about which we have never made any constrainig assumptions). More specifically, the masses and decay widths of the dark hadronic resonances depend on all of the parameters of the strongly interacting Lagrangian - on the values of the coupling $g_{\text {dark }}$ and the dark-quark masses $m_{q_{i}^{\text {dark }}}, m_{H}^{\text {dark }}=m_{H}^{\text {dark }}\left(g, m_{q_{1}}, m_{q_{2}}, \ldots\right)$. Thus, a strongly-coupled theory need not imply large decay widths between the composite particles.

This may seem surprising because QCD (the particular realization of strong interaction in the visible sector) has a Lagrangian with parameter values that lead to rapid intra-spectrum decays. However, this need not necessarily hold for the entire parameter region. In particular, there have

\footnotetext{
${ }^{1}$ We thank Luka Leskovec for important discussions on this point. Further details can be found in Ref. [17].
} 
been several recent start-of-the-art lattice-QCD calculations — all reviewed in in Ref. [13] — in which the light quark masses are set to values that are higher than those observed in the visible sector. Interestingly, a global picture is emerging from these studies, namely that there exists a region of parameter space in which hadronic resonances appear as stable bound states because their decay channels are kinematically forbidden. Of course, whether this property generalizes to all strongly interacting theories is unclear. However, there are studies that support this. For example, Ref. [15] provides an example in which the mass of the $\rho$ particle mass can lie either below or above the $2 m_{\pi}$ threshold (see Fig. 2). Another very interesting study is the investigation of the $\rho$ in $S U(2)$ strongly-coupled gauge theories presented in Ref. [16].

Acknowledgments: We are happy to thank Luka Leskovec for very informative discussions. We also thank the conference organizers for their invitation to come to Maigh Nuad and deliver this talk. The research activities of KRD, FH, and SS were supported in part by U.S. Department of Energy Grant DE-SC0009913; those of KRD were also supported in part by the U.S. National Science Foundation through its employee IR/D program. The opinions and conclusions expressed herein are those of the authors, and do not represent any funding agencies.

\section{References}

[1] K. R. Dienes, F. Huang, S. Su and B. Thomas, Phys. Rev. D 95, 043526 (2017) [arXiv:1610.04112 [hep-ph]].

[2] K. R. Dienes and B. Thomas, Phys. Rev. D 85, 083523 (2012) [arXiv:1106.4546 [hep-ph]].

[3] K. R. Dienes and B. Thomas, Phys. Rev. D 85, 083524 (2012) [arXiv:1107.0721 [hep-ph]].

[4] K. R. Dienes, S. Su and B. Thomas, Phys. Rev. D 86, 054008 (2012) [arXiv:1204.4183 [hep-ph]].

[5] K. R. Dienes, S. Su and B. Thomas, Phys. Rev. D 91, no. 5, 054002 (2015) [arXiv:1407.2606 [hep-ph]].

[6] K. R. Dienes, J. Kumar and B. Thomas, Phys. Rev. D 86, 055016 (2012) [arXiv:1208.0336 [hep-ph]].

[7] K. R. Dienes, J. Kumar and B. Thomas, Phys. Rev. D 88, no. 10, 103509 (2013) [arXiv:1306.2959 [hep-ph]].

[8] K. K. Boddy, K. R. Dienes, D. Kim, J. Kumar, J. C. Park and B. Thomas, arXiv:1606.07440 [hep-ph].

[9] K. K. Boddy, K. R. Dienes, D. Kim, J. Kumar, J. C. Park and B. Thomas, arXiv:1609.09104 [hep-ph].

[10] K. R. Dienes and B. Thomas, Phys. Rev. D 86, 055013 (2012) [arXiv:1203.1923 [hep-ph]].

[11] K. R. Dienes, J. Fennick, J. Kumar and B. Thomas, to appear.

[12] K. R. Dienes, J. Fennick, J. Kumar and B. Thomas, Phys. Rev. D 93, 083506 (2016) [arXiv:1601.05094 [hep-ph]].

[13] R. A. Briceno, J. J. Dudek and R. D. Young, Rev. Mod. Phys. 90, 025001 (2018) [arXiv:1706.06223 [hep-lat]].

[14] R. A. Briceno, EPJ Web Conf. 175, 01016 (2018).

[15] T. Appelquist et al., Phys. Rev. D 93, 114514 (2016) [arXiv:1601.04027 [hep-lat]].

[16] T. Janowski, V. Drach and C. Pica, PoS LATTICE 2016, 214 (2016) [arXiv:1611.09195 [hep-lat]].

[17] K. R. Dienes, F. Huang, L. Leskovec, S. Su and B. Thomas, in preparation. 